Exp. Anim. 49(4), 267-274, 2000

\title{
APA Hamster Model for Diabetic Atherosclerosis. 2. Analysis of Lipids and Lipoproteins
}

\author{
J un YAMANOUCHI, Atsushi TAKATORI, Shin-ichi ITAGAKI, \\ Seiji KAWAMURA, and Yasuhiro YOSHIKAWA
}

Department of Biomedical Science, Graduate School of Agricultural and Life Sciences, The University of Tokyo, Yayoi 1-1-1, Bunkyo-ku, Tokyo 113-8657, Japan

\begin{abstract}
Syrian hamsters of the APA strain (APA hamsters) have recently been shown to have atheromatous lesions in the aortic arches under diabetic condition induced by a single injection of streptozotocin (SZ). In that model, fatty streaks, which are the initial lesions of atherogenesis, develop by 6 weeks after the injection (WAI). In this study, we evaluated plasma lipid concentrations and lipoprotein profiles in diabetic APA hamsters at 6 WAI to reveal the early stage of atherogenesis clinicopathologically. As a result, by biochemical analysis, hyperglycemic APA hamsters showed signs of hypercholesterolemia and hypertriglyceridemia. Low-density lipoprotein (LDL) cholesterol significantly increased, but high-density lipoprotein (HDL) cholesterol significantly decreased. Agarose gel electrophoresis showed an obvious increase in the fractions of chylomicron, $L D L$ and abnormal lipoprotein. Plasma LDL in diabetic animals was in a state more susceptible to oxidization. In addition, a significant increase in glycated LDL was also found in the diabetic animals by enzyme linked immunosorbent assay (ELISA). Moreover, lipid peroxidation product (4-hydroxynonenal (4 HNE))-adducted proteins and advanced glycation end-products (AGE) were immunohistochemically detected in the foam cells of the fatty streaks. These results revealed that diabetic APA hamsters had hyperlipidemia characterized by increases in chylomicron, $L D L$ and abnormal lipoprotein, and suggested that oxidized $L D L$ and/or glycated $L D L$ might be actively uptaken by macrophages and play an important role in the initial stage of atherogenesis.
\end{abstract}

Key words: APA hamster, diabetic atherosclerosis, lipoprotein, modified LDL

\section{Introduction}

Diabetes is known to be often accompanied by hyperlipidemia, which is most closely associated with atherosclerosis. In many clinical studies on patients with atherosclerosis, a high level of blood cholesterol or triglyceride, a high level of low-density lipoprotein (LDL) or triglyceride-rich lipoproteins (very low-density lipoproteins (VLDL) and chylomicron), and a low level of high density lipoprotein (HDL) are described as risk markers for atherosclerosis $[14,15,26,27,37$, 54].

(Received 8 November 1999 / Accepted 13 April 2000)

Address corresponding: Y. Yoshikawa, Department of Biomedical Science, Graduate School of Agricultural and Life Sciences, The University of Tokyo, Yayoi 1-1-1, Bunkyo-ku, Tokyo 113-8657, Japan 
Among various risk factors involved in atherogenesis, LDL has especially been studied and reproted to play a crucial role in the formation of foam cells (FCs). The accumulation of FCs characterizes fatty streaks which are the initial atheromatous lesions. FC formation results from uptake of modified forms of LDL but not native LDL mainly by macrophages $(\mathrm{M} \phi \mathrm{s})$. Most well known modified LDL is oxidized LDL (ox-LDL) $[17,28,38]$, which has been detected in blood and atheromatous lesions of humans and laboratory animals $[8,22,60,64]$.

In addition to ox-LDL, glycated or glyco-oxidized LDL in diabetic patients has been considered to explain rapid and severe progression of atherosclerosis $[56,59]$. In this connection, advanced glycation end-products (AGE), which are the irreversible last-stage products in non-enzymatic glycation, have drawn much attention recently. A significant increase in AGE has been detected in the blood of diabetic patients [31, 63] and AGE deposition in atherosclerotic lesions has been detected in patients with diabetes [25, 31, 34].

In Syrian hamsters, apolipoprotein (apo) B-48 containing lipoproteins is exclusively of intestinal origin and apoB-100 is exclusively of hepatic origin, which is analogous to the condition in humans but not in mice or rats [4, 13]. Syrian hamsters of the APA strain (APA hamsters) have recently been shown to have atheromatous lesions in the aortic arches under diabetic conditions induced by a single injection of streptozotocin (SZ) [62]. In that model, fatty streaks, which are the initial lesions in atherogenesis, develop until 6 weeks after the injection (WAI). In this study, we therefore evaluated plasma lipid concentrations and lipoprotein profiles in diabetic APA hamsters at $6 \mathrm{WAI}$ to reveal the early stage of atherogenesis clinicopathologically. We also examined depositions of oxidized deoxynucleic acids (DNA), lipid peroxidation product-adducted proteins and AGE in the aortic walls to show some evidence of oxidation and glycation of lipoproteins.

\section{Materials and Methods}

\section{Animals}

We examined 4 hyperglycemic male APA hamsters which were once injected at the age of 10 weeks intraperitoneally with $40 \mathrm{mg} / \mathrm{kg}$ body weight of SZ (Sigma
Chemical Co., St Louis, USA) dissolved in $2 \mathrm{~m} l$ of $0.1 \mathrm{M}$ citrate buffer (CB) ( $\mathrm{pH} 4.5)$, and the same number of animals injected with the same volume of $\mathrm{CB}$ only were used as controls. The former were called the SZ group and the latter the CB group. The animals were purchased from Japan SLC Inc. (Shizuoka, Japan) and maintained under controlled conditions (temperature: $24 \pm 2^{\circ} \mathrm{C}$; dark-light cycle: $12-12 \mathrm{hr}$ ) in plastic cages with sterilized wood shavings for bedding, and fed a commercial diet, CMF (Oriental Yeast Co. Ltd., Tokyo, Japan) with tap water ad libitum. This experiment was approved by the Animal Care and Use Committee of Graduate School of Agricultural and Life Sciences, The University of Tokyo.

\section{Sampling}

At 6 WAI, each animal was weighed and killed by collecting blood from the vena cava under ether anaesthesia after overnight (14-16 hr) fasting. Immediately after exsanguination, the aorta was taken from each animal and fixed in 4\% paraformaldehyde in $0.01 \mathrm{M}$ phosphate buffered saline (PBS). Fresh plasma samples were separated from $0.1 \%$ ethylenediaminetetraacetic acid (EDTA) containing blood samples and added to the mixture including $0.02 \%$ sodium azide $\left(\mathrm{NaN}_{3}\right), 1$ $\mathrm{mM}$ phenylmethylsulfonyl fluoride (PMSF), $0.03 \%$ benzamidine, $10 \mu \mathrm{g} / \mathrm{m} l$ gentamycin and $5 \mathrm{mM}$ butylated hydroxytoluene (BHT) for storage at $4^{\circ} \mathrm{C}$.

\section{Plasma biochemistry}

Plasma samples were subjected to the measurement of glucose, triglyceride, total cholesterol, and HDL cholesterol with commercial test kits (Wako Pure Chemical Industries Inc., Osaka, Japan). HDL cholesterol was measured after removing apolipoprotein B-containing particles by the magnesium/phosphotungstic acid precipitation method [57, 58]. LDL cholesterol levels were quantified with LDL Cholesterol Kit (Sigma Diagnostic, Inc., St Louis, USA) and the above mentioned test kit for total cholesterol [45]; after the removal of HDL and VLDL by incubating with latex beads coated with goat polyclonal antibodies to human apolipoproteins and filtrating, LDL cholesterol in the filtrate was determined. Since plasma samples in the SZ group were milky white, the absorbance of plasma in each reaction buffer without an enzyme was measured as a background, except for measuring $\mathrm{HDL}$ and LDL cholesterol. Plasma 
samples in the SZ group were also 2 or 4 fold diluted with saline so that values might be within the limits in the manufacturer's guide. Student's $t$-test was used for statistical analysis.

\section{Lipoprotein electrophoresis}

Fractions of plasma lipoproteins were analyzed by agarose gel electrophoresis [58]. Aliquots of $1 \mu \mathrm{l}$ plasma in the CB group and $1 \mu l$ twice-diluted plasma with saline in the SZ group were used. Electrophoresis was carried out on $1 \%$ agarose gel films (Agasheet-L, Wako) with a horizontal apparatus (Submerge-Agarose-System, Atto Corporation, Tokyo, Japan) at 90 volts for $45 \mathrm{~min}$, followed by staining with Fat Red 7B, on the day of sampling and after storage of the plasma at $4^{\circ} \mathrm{C}$ for 2 days. Electrophoresis patterns of lipoproteins were also scanned by means of a computerized densitometry system.

\section{Enzyme-linked immunosorbent assay (ELISA)}

Glycated LDL levels in plasma samples were measured with the ELISA kit (Exocell, Inc., Philadelphia, USA), according to the manufacturer's guide. Twenty $\mu l$ of plasma was mixed with $800 \mu l$ of a diluent reagent and added to a microplate pre-coated with glycated LDL. The plate was then incubated with a mouse monoclonal antibody to human glycated LDL for $1 \mathrm{hr}$, washed, and incubated with horseradish peroxidase (HRP)-conjugated anti-mouse immunoglobulin $\mathrm{G}$ goat antibody for $1 \mathrm{hr}$. Color developing was performed with tetramethylbenzidine (TMB) for $20 \mathrm{~min}$. The standard curve was drawn by plotting 5 points: 1 , 2, 4, 8 and 16-fold diluted standard glycated LDL. Statistical analysis was done with Student's $t$-test.

\section{Immunohistochemistry}

Four $\mu \mathrm{m}$ thick paraffin sections of the longitudinal aortic arches were stained by the indirect immunohistochemical method for 8-hydroxydeoxyguanosine (8-OHdG), 4-hydroxynonenal (4 HNE) and AGE. 8OHdG is an oxidized product of 2-deoxyguanosine and is a marker for oxidative damage to DNA. 4-HNE is an oxidized product from polyunsaturated fatty acids (n-6), such as arachidonic acid, and reacts with amino acid residues of proteins, leading to the formation of stable Michael adducts. Both these oxidized markers can be confidencially detected on the usual paraffin sections without special antioxidative treatment $[9,21,24,52$, 53]. After treated with $0.1 \%$ trypsin only for $8-\mathrm{OHdG}$, with $0.3 \%$ hydrogen peroxide in methanol, and with $1 \%$ bovine serum albmin, the sections were treated with mouse monoclonal antibodies to 8-OHdG (NOF Corporation, Tokyo, Japan; 1:100 dilution), 4-HNE (NOF Corporation, 1:1000 dilution) or AGE (Kumamoto Immunochemical Laboratory Co., Ltd., Kumamoto, Japan, 1:300 dilution), followed by biotinylated rabbit antibody to mouse immunoglobulins (DAKO A/S, Glostrup, Denmark) and streptavidin-biotin peroxidase complex (StreptABComplex/HRP kit, DAKO). Visualization was performed with 3,3' diaminobenzidine (DAB).

\section{Results}

\section{Plasma biochemistry (Table 1)}

Plasma glucose levels in the SZ group were 3-fold higher than those in the CB group (statistical significance: $\mathrm{p}<0.001$ ). Triglyceride levels were about 10 -fold higher and total cholesterol levels were about 4.5 -fold higher in the SZ group than in the CB group (statistical significance: $p<0.01$ and $p<0.001$, respectively). LDL cholesterol levels in the SZ group were about 3-fold higher than those in the CB group (statistical significance: $\mathrm{p}<0.01)$. In contrast, HDL cholesterol levels in the SZ group were significantly lower than in the CB group (statistical significance: $\mathrm{p}<0.01$ ).

\section{Lipoprotein electrophoresis}

The fractions of chylomicron and LDL significantly increased in the SZ group, as compared with the $\mathrm{CB}$ group (Figs. 1a, b). The fractions of VLDL in the SZ group had broad bands but no clear peaks and (Figs. $1 \mathrm{a}, \mathrm{b})$, and they increased a little, as compared to the CB group (Fig. 1b). Abnormal fractions also appeared between the chylomicron and the LDL fractions in the SZ group (Figs. 1a, b). The HDL fractions were not detected in either the CB or SZ group.

Moreover, the relative mobility of the LDL fractions in the SZ group increased as compared to that in the $\mathrm{CB}$ group after storage of the plasma samples for 2 days at $4^{\circ} \mathrm{C}$ (Figs. 1c, d), suggesting that oxidation of LDL in the SZ group progressed in spite of the addition of antioxidants. 

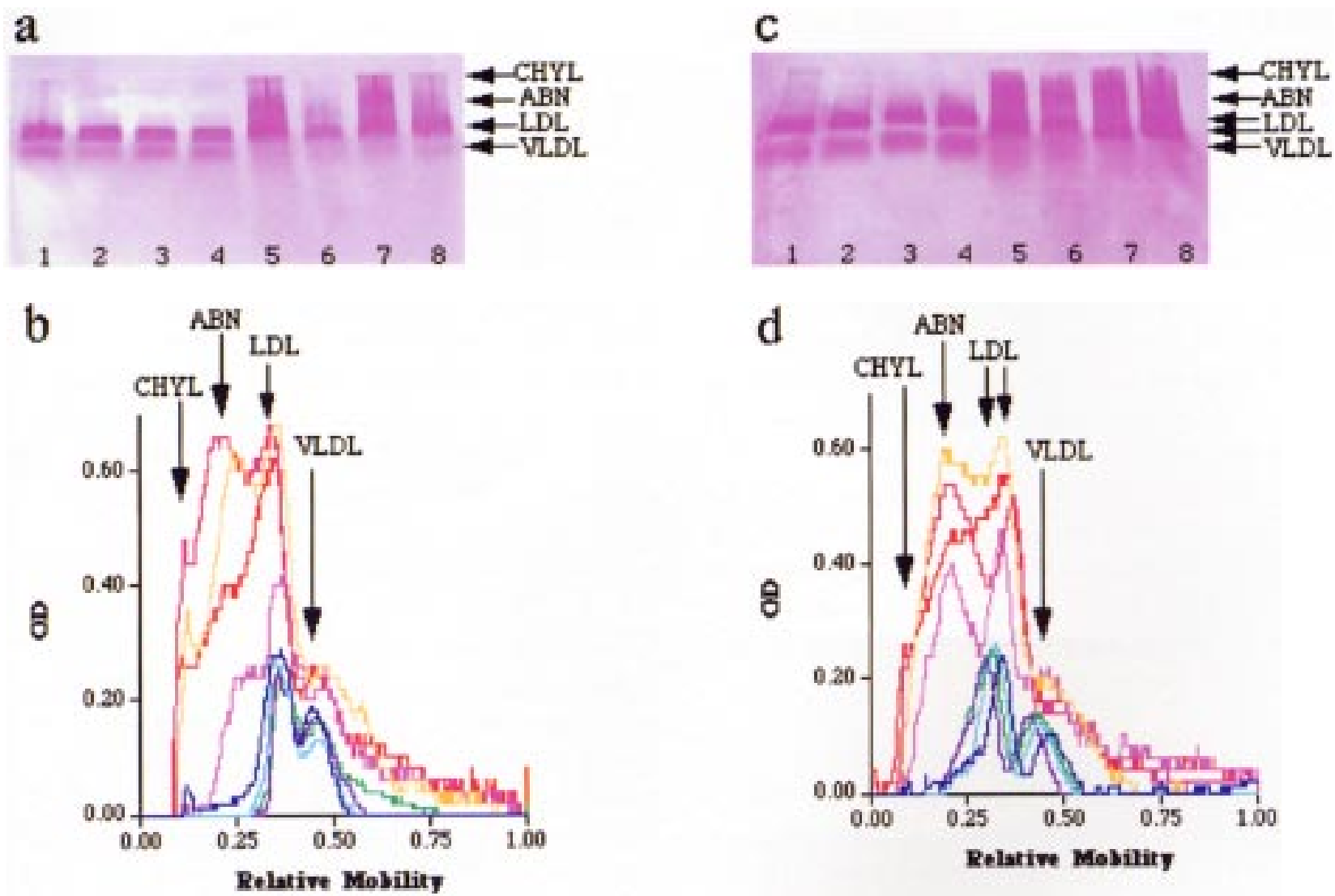

Fig. 1. Lipoprotein electrophoresis on agarose gels on the day of sampling $(\mathrm{a}, \mathrm{b})$ and after storage at $4^{\circ} \mathrm{C}$ for 2 days (c, d). Lanes $1-4$ : the CB group; lanes 5-8: the SZ group. CHYL: chylomicron; LDL: low-density lipoprotein; VLDL: very low-density lipoprotein; ABN: abnormal lipoprotein. In $\mathrm{b}$ and d, dark-blue line; lane 1; blue line: lane 2; violet line: lane 3; green line: lane 4; yellow line: lane 5; pink line: lane 6; magenta line: lane 7; red line: lane 8. Note that the samples in the SZ group were twice-diluted with saline (a, c) and scanned data in the SZ group were twice-multiplied data (b, d).

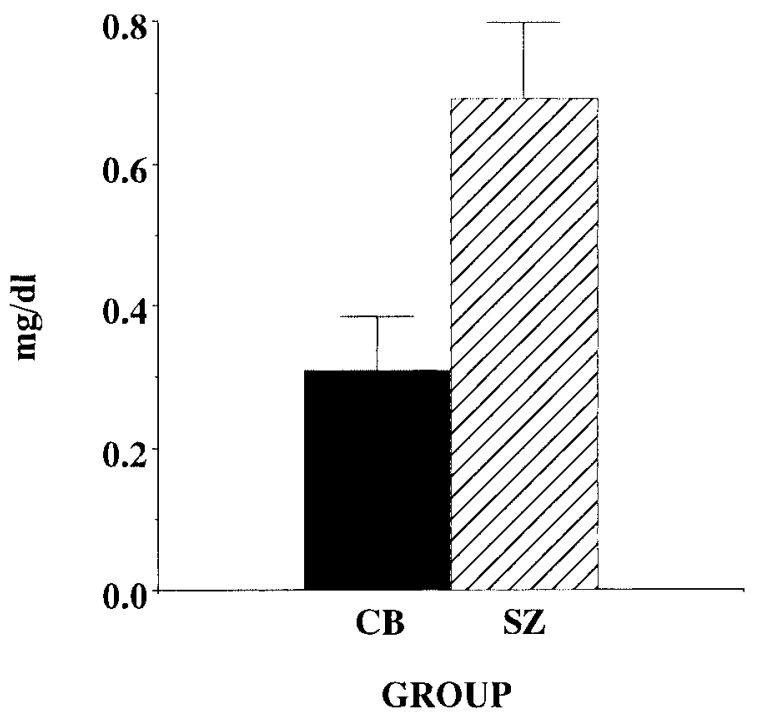

\section{Glycated LDL levels}

A significant increase in plasma glycated LDL levels was seen in the SZ group, as compared with in the $\mathrm{CB}$ group (Fig. 2).

\section{Immunohistochemical findings}

In the SZ group, 8-OHdG was clearly detected in the nuclei of FCs of the fatty streaks (Fig. 3a) and in some endothelial cells (ECs), and weakly detected in smooth muscle cells. 4-HNE-adducted proteins (Fig. 3b) and AGE (Fig. 3c) are seen mainly in FCs of the fatty streaks and sparsely in the media. In the CB group, 8 -OHdG, 4-HNE and AGE were very rarely found only in the media (data not shown).

Fig. 2. Plasma glycated LDL levels in the CB and SZ group. Values are expressed as means \pm SDs $[\mathrm{mg} / \mathrm{d} l]$. Statistical analysis was performed with Student's $t$-test; $\mathrm{p}<0.01$. 

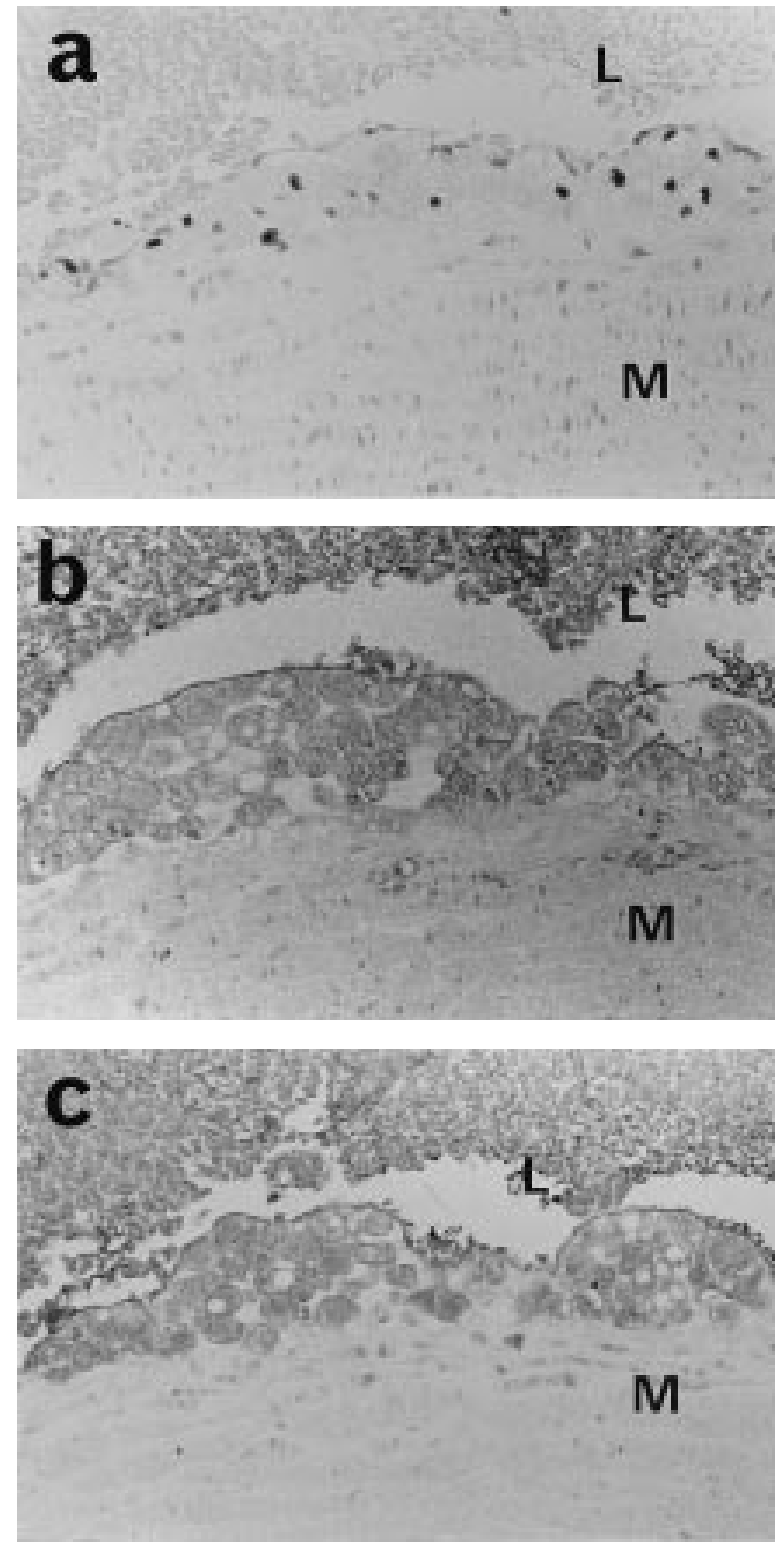

Fig. 3. Immunohistochemical detection of $8-\mathrm{OHdG}$ (a), 4-HNE (b) and AGE (c) on the aortic arches of APA hamsters in the SZ group. L: lumen; M: media. 8-OHdG (a) is clearly found in the nuclei of foam cells (FCs) and some endothelial cells in a fatty streak, and is weakly detected in the nuclei of smooth muscle cells. 4-HNE-adducted proteins (b) and AGE (c) are seen mainly in FCs in the fatty streaks and sparsely in the media. $\times 220$.

\section{Discussion}

We have recently demonstrated that the fatty streaks, which are the initial atheromatous lesions, developed in diabetic APA hamsters until 6 WAI [62]. In this

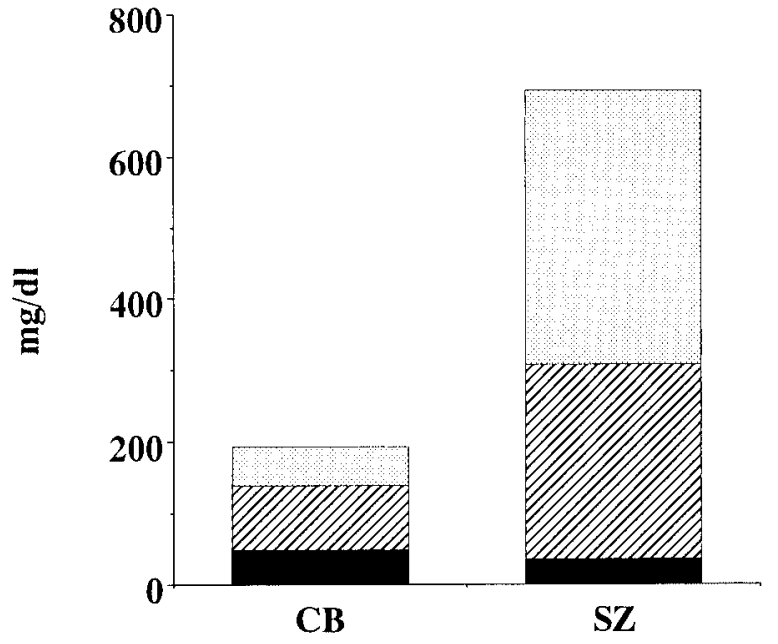

GROUP

Fig. 4. Changes in cholesterol levels of lipoproteins at in the CB and SZ groups. Black bars: high-density lipoprotein; line bars: low-density lipoprotein; dot bars: other lipoproteins.

study, we evaluated plasma lipid concentrations and lipoprotein profiles in diabetic APA hamsters at 6 WAI to reveal the early stage of atherogenesis clinicopathologically. As a result, diabetic APA hamsters showed signs of hypercholesterolemia and hypertriglyceridemia, which are usual complications in diabetic patients. Biochemical analysis of the cholesterol of lipoproteins revealed a significant increase in LDL and a decrease in HDL, which are general markers for atherosclerosis in humans [25, 37, 54]. Nevertheless, an increase in cholesterol levels of nonLDL and non-HDL lipoproteins was more conspicuous after subtracting LDL and HDL from total cholesterol levels (Fig. 4). In fact, lipoprotein electrophoresis revealed obvious increases in chylomicron and abnormal fractions as well as the LDL fraction. It is noted that apo B-48 containing lipoproteins are exclusively of intestinal origin in Syrian hamsters [4, 13] and the chyromicron level was high even though blood was collected during overnight fasting in this model. The lipoprotein changes in this model could not simply be classified as hyperlipidemia in human patients, and further studies are needed to clarify the mechanisms.

The abnormal fraction which appeared between the chylomicron and LDL fractions in lipoprotein electrophoresis might be suspected of being lipoprotein $\mathrm{X}$ 
(Lp-X) [47], which has been reported in patients with cholestatic liver diseases [11,33, 44], patients with a deficiency of lecithin/cholesterol acyltransferase (LCAT) $[16,36,48]$ and during intravenous infusion of fat emulsions $[2,50,51] . \mathrm{Lp}-\mathrm{X}$ is a bilayer vesicle of phospholipids (51-66\%) and free cholesterol (22-30\%) and contains a small amount of proteins essentially including albumin and apoCs but also including apoE and apoAI $[2,16,33,35,36,39,44,48] . \mathrm{Lp}-\mathrm{X}$ is postulated to be related to biliary lipid vesicles, but the mechanism of Lp-X generation remains unclear. The role of Lp-X in atherogenesis has also not been clarified; Lynn et al. [29] have only reported that Lp-X enhanced FC formation in rat $\mathrm{M} \phi \mathrm{s}$, whereas other reports have demonstrated that $\mathrm{Lp}-\mathrm{X}$ did not enhance [42, 43] but rather suppressed [1, 2] FC formation. More studies are needed to reveal whether the abnormal lipoprotein seen in this study is Lp-X, and its pathological significance in this model.

Agarose gel electrophoresis revealed advanced oxidization of LDL in the SZ group by increasing the relative mobility of the LDL fraction $[6,18,19,41$, $61]$, though a clear difference between the plasma oxLDL concentrations in the SZ group and those in the CB group could not be detected by dot blot analysis (data not shown). The susceptibility of LDL to oxidation has been reported to have a significant relation to progression of atherosclerosis in humans [3, 40], and more attention has been paid to the oxidizability of LDL from the aspect of local oxidization in the arterial walls $[5,46,49,54]$. Therefore, when there would be excessive LDL in blood, LDL might stay longer in the arterial wall, where it could be oxidatively modified because of its high susceptibility to oxidization. Moreover, ox-LDL in the arterial wall would actively be uptaken by invading $\mathrm{M} \phi \mathrm{s}$, enhancing FC formation. In fact, lipid peroxidation product (4-HNE)-adducted proteins and oxidized DNA (8-OHdG) were immunohistochemically detected in the fatty streaks in diabetic APA hamsters, giving evidence of oxidative stress in the aortic walls of those animals.

Moreover, glycated LDL, whose levels significantly increased in the SZ group, has also been shown to be very sensitive to oxidation [7, 23, 32]. It has been suggested that glyco-oxidized LDL is more rapidly uptaken by $M \phi$ s than merely oxidized LDL. In fact, AGE deposition in the atheromatous lesions was im- munohistochemically detected in FCs in this study. In diabetic complications, AGE is especially important and has been supposed to be associated with more rapid and severe development of atheromatous lesions in diabetic patients than in non-diabetic people $[10,12,20$, 30].

In diabetic APA hamsters, hyperlipidemia and hyperlipoproteinemia are characterized by increases in chylomicron, LDL and abnormal lipoprotein. Glycated and/or oxidized LDL is considered to be actively uptaken by $M \phi$ s and to play an important role in the initial stage of atherogenesis.

\section{Acknowledgment}

This study was supported by Research Fellowships of the Japan Society for the Promotion of Science and by Grants-in-Aid for Scientific Research from the Ministry of Education, Science and Culture of Japan.

\section{References}

1. Abe, M., Kawano, M., Tashiro, T., Yamamori, H., Takagi, K., Morishima, Y., Shirai, K., Saitou, Y., and Nakajima, N. 1997. Catabolism of lipoprotein-X induced by infusion of 10\% fat emulsion. Nutrition 13: 417-421.

2. Abe, M., Tashiro, T., Mashima, Y., Yamamori, H., Nishizawa, M., Sanada, M., and Okui, K. 1991. [Catabolism of lipoprotein-X (Lp-X) induced by infusion of $10 \%$ intralipid]. Nippon Geka Gakkai Zasshi 92: 1678-1685.

3. Andrews, B., Burnand, K., Paganga, G., Browse, N., Rice, E.C., Sommerville, K., Leake, D., and Taub, N. 1995. Oxidisability of low density lipoproteins in patients with carotid or femoral artery atherosclerosis. Atherosclerosis 112: 77-84.

4. Arbeeny, C.M., Meyers, D.S., Bergquist, K.E., and Gregg, R.E. 1992. Inhibition of fatty acid synthesis decreases very low density lipoprotein secretion in the hamster. J. Lipid Res. 33: 843-851.

5. Aviram, M., Maor, I., Keidar, S., Hayek, T., Oiknine, J., Bar, E.Y., Adler, Z., Kertzman, V., and Milo, S. 1995. Lesioned low density lipoprotein in atherosclerotic apolipoprotein E- deficient transgenic mice and in humans is oxidized and aggregated. Biochem. Biophys. Res. Commun. 216: 501-513.

6. Bolton, E. J., Jessup, W., Stanley, K. K., and Dean, R. T. 1994. Enhanced LDL oxidation by murine macrophage foam cells and their failure to secrete nitric oxide. Atherosclerosis 106: 213-223.

7. Bowie, A., Owens, D., Collins, P., Johnson, A., and Tomkin, G.H. 1993. Glycosylated low density lipoprotein is more sensitive to oxidation: implications for the diabetic patient? 
Atherosclerosis 102: 63-67.

8. Boyd, H.C., Gown, A.M., Wolfbauer, G., and Chait, A. 1989. Direct evidence for a protein recognized by a monoclonal antibody against oxidatively modified LDL in atherosclerotic lesions from a Watanabe heritable hyperlipidemic rabbit. Am. J. Pathol. 135: 815-825.

9. Casasco, A., Calligaro, A., Casasco, M., Tateo, S., IcaroCornaglia, A., Reguzzoni, M., and Farina, A. 1997. Immunohistochemical localization of lipoperoxidation products in normal human placenta. Placenta 18: 249-253.

10. Chappey, O., Dosquet, C., Wautier, M.P., and Wautier, J.L. 1997. Advanced glycation end products, oxidant stress and vascular lesions. Eur. J. Clin. Invest. 27: 97-108.

11. Chisholm, J.W. and Dolphin, P.J. 1996. Abnormal lipoproteins in the ANIT-treated rat: a transient and reversible animal model of intrahepatic cholestasis. J. Lipid. Res. 37: 1086-1098.

12. Dobrian, A. and Simionescu, M. 1995. Irreversibly glycated albumin alters the physico-chemical characteristics of low density lipoproteins of normal and diabetic subjects. Biochim. Biophys. Acta. 1270: 26-35.

13. Ebara, T., Hirano, T., Mamo, J.C.L., Sakamaki, R., Furukawa, S., Nagano, S., and Takahashi, T. 1994. Hyperlipidemia in streptozotocin-diabetic hamsters as a model for human insulin-deficient diabetes: comparison to streptozotocin-diabetic rats. Metabolism 43: 299-305.

14. Ginsberg, H.N. 1996. Diabetic dyslipidemia: basic mechanisms underlying the common hypertriglyceridemia and low HDL cholesterol levels. Diabetes S27-S30.

15. Gotto, A.J. 1992. Hypertriglyceridemia: risks and perspectives. Am. J. Cardiol. 70: 19H-25H.

16. Guerin, M., Dolphin, P.J., and Chapman, M.J. 1993. Familial lecithin:cholesterol acyltransferase deficiency: further resolution of lipoprotein particle heterogeneity in the low density interval. Atherosclerosis 104: 195-212.

17. Hamilton, C.A. 1997. Low-density lipoprotein and oxidised low-density lipoprotein: their role in the development of atherosclerosis. Pharmacol. Ther. 74: 55-72.

18. Harduin, P., Tailleux, A., Lestavel, S., Clavey, V., Fruchart, J-C., and Fievet, C. 1995. Immunological and functional properties of in vitro oxidized low density lipoprotein. $J$. Lipid Res. 36: 919-930.

19. Heinecke, J. W., Kawamura, M., Suzuki, L., and Chait, A. 1993. Oxidation of low density lipoprotein by thiols: superoxide-dependent and -independent mechanisms. $J$. Lipid Res. 34: 2051-2061.

20. Hiraga, T. 1994. [Glycosylated lipoprotein]. Nippon Rinsho 52: 3096-3099.

21. Ihara, Y., Toyokuni, S., Uchida, K., Odaka, H., Tanaka, T., Ikeda, H., Hiai, H., Seino, Y., and Yamada, Y. 1999. Hyperglycemia causes oxidative stress in pancreatic betacells of GK rats, a model of type 2 diabetes. Diabetes 48 : 927-932.

22. Kanazawa, T., Uemura, T., Osanai, T., Fukushi, Y., Imura, T., Oike, Y., Onodera, K., Akasaka, K., Okubo, K., and Takahashi, S. 1994. Plasma peroxidized low-density lipoprotein with hydroperoxidized cholesteryl linoleates estimated in patients with familial hypercholesterolemia.
Pathobiology 62: 269-282.

23. Kobayashi, K., Watanabe, J., Umeda, F., and Nawata, H. 1995. Glycation accelerates the oxidation of low density lipoprotein by copper ions. Endocr. J. 42: 461-465.

24. Kondo, S., Toyokuni, S., Iwasa, Y., Tanaka, T., Onodera, H., Hiai, H., and Imamura, M. 1999. Persistent oxidative stress in human colorectal carcinoma, but not in adenoma. Free Radic. Biol. Med. 27: 401-410.

25. Kume, S., Takeya, M., Mori, T., Araki, N., Suzuki, H., Horiuchi, S., Kodama, T., Miyauchi, Y., and Takahashi, K. 1995. Immunohistochemical and ultrastructural detection of advanced glycation end products in atherosclerotic lesions of human aorta with a novel specific monoclonal antibody. Am. J. Pathol. 147: 654-667.

26. Laakso, M. 1996. Lipids and lipoproteins as risk factors for coronary heart disease in non insulin-dependent diabetes mellitus. Ann. Med. 28: 341-345.

27. Lamarche, B. and Lewis, G.F. 1998. Atherosclerosis prevention for the next decade: Risk assessment beyond low density lipoprotein cholesterol. Can. J. Cardiol. 14: 841-851.

28. Lopes, V.M. and Virella, G. 1996. Modified lipoproteins, cytokines and macrovascular disease in non-insulindependent diabetes mellitus. Ann. Med. 28: 347-354.

29. Lynn, E.G. and Karmin, O. 1997. Role of lipoprotein-X in foam cell formation and rat mesangial cell proliferation. Clin. Exp. Pharmacol. Physiol. 24: 973-975.

30. Lyons, T.J. and Jenkins, A.J. 1997. Lipoprotein glycation and its metabolic consequences. Curr. Opin. Lipidol. 8: 174-180.

31. Makita, Z., Vlassara, H., Cerami, A., and Bucala, R. 1992. Immunochemical detection of advanced glycosylation end products in vivo. J. Biol. Chem. 267: 5133-5138.

32. Maziere, C., Auclair, M., Rose, R.F., Leflon, P., and Maziere, J.C. 1995. Glucose-enriched medium enhances cell-mediated low density lipoprotein peroxidation. FEBS Lett. 363: 277-279.

33. Miller, J.P. 1990. Dyslipoproteinaemia of liver disease. Baillieres Clin. Endocrinol. Metab. 4: 807-832.

34. Nakamura, Y., Horii, Y., Nishino, T., Shiiki, H., Sakaguchi, Y., Kagoshima, T., Dohi, K., Makita, Z., Vlassara, H., and Bucala, R. 1993. Immunohistochemical localization of advanced glycosylation end products in coronary atheroma and cardiac tissue in diabetes mellitus. Am. J. Pathol. 143: 1649-1656.

35. Narayanan, S. 1984. Biochemistry and clinical relevance of lipoprotein X. Ann. Clin. Lab. Sci. 14: 371-374.

36. O, K. and Frohlich, J. 1995. Role of lecithin:cholesterol acyltransferase and apolipoprotein A-I in cholesterol esterification in lipoprotein-X in vitro. J. Lipid. Res. 36: 2344-2354.

37. Okubo, M. and Murase, T. 1996. Hypertriglyceridemia and low HDL cholesterol in Japanese patients with NIDDM. Diabetes S123-S125.

38. Parthasarathy, S., Steinberg, D., and Witztum, J.L. 1992. The role of oxidized low-density lipoproteins in the pathogenesis of atherosclerosis. Annu. Rev. Med. 43: 219225. 
39. Patsch, J.R., Kirk, C.A., Gotto, A.M. Jr., and Morrissett, J.D. 1977. Isolation, chemical characterization, and biophysical properties of three different abnormal lipoproteins LP-X1, LP-X2, LP-X3. J. Biol. Chem. 232: 2113-2120.

40. Regnstrom, J., Nilsson, J., Tornvall, P., Landou, C., and Hamsten, A. 1992. Susceptibility to low-density lipoprotein oxidation and coronary atherosclerosis in man. Lancet 339: 1183-1186.

41. Rikitake, Y., Hirata, K., Kawashima, S., Akita, H., and Yokoyama, M. 1998. Inhibitory effect of inducible type nitric oxide synthase on oxidative modification of low density lipoprotein by vascular smooth muscle cells. Atherosclerosis 136: 51-57.

42. Rotheneder, M. and Kostner, G.M. 1986. Cholesterol esterification in mouse peritoneal macrophages in the presence of pathological human plasma lipoproteins. Biol. Chem. Hoppe. Seyler 367: 1219-1222.

43. Rotheneder, M., Krempler, F., and Kostner, G.M. 1988. Interaction of various lipoproteins from normal and dyslipoproteinemic plasma with mouse peritoneal macrophages. Ann. Biol. Clin. (Paris) 46: 30-34.

44. Sabesin, S.M. 1982. Cholestatic lipoproteins - their pathogenesis and significance. Gastroenterology 83: 704709.

45. Schectman, G., Patsches, M., and Sasse, E.A. 1996. Variability in cholesterol measurements: Comparison of calculated and direct LDL cholesterol determinations. Clin. Chem. 42: 732-737.

46. Schwenke, D.C. 1995. Selective increase in cholesterol at atherosclerosis-susceptible aortic sites after short-term cholesterol feeding. Arterioscler. Thromb. Vasc. Biol. 15: 1928-1937.

47. Seidel, D., Alaupovic, P., and Furman, R.H. 1969. A lipoprotein characterizing obstructive jaundice. I. Method for quantitative separation and identification of lipoproteins in jaundiced subjects. J. Clin. Invest. 48: 1211-1223.

48. Seidel, D., Gjone, E., Blomhoff, J.P., and Geisen, H.P. 1974. Plasma lipoproteins in patients with familial plasma lecithin: Cholesterol acyltransferase (LCAT) deficiency—studies on the apolipoprotein composition of isolated fractions with identification of Lp-X. Horm. Metab. Res. Suppl. 4: 6-11.

49. Suzuki, H., Kurihara, Y., Takeya, M., Kamada, N., Kataoka, M., Jishage, K., Ueda, O., Sakaguchi, H., Higashi, T., Suzuki, T., Takashima, Y., Kawabe, Y., Cynshi, O., Wada, Y., Honda, M., Kurihara, H., Aburatani, H., Doi, T., Matsumoto, A., Azuma, S., Noda, T., Toyoda, Y., Itakura, H., Yazaki, Y., Horiuchi,S., Takahashi, K., Kruijs, J.K., van Berkel, T.J.C., Steinbrecher, V.P., Ishibashi, S., Maeda, N., Gordon, S., and Kodama, T. 1997. A role for macrophage scavenger receptors in atherosclerosis and susceptibility to infection. Nature 386: 292-296.

50. Tashiro, T., Mashima, Y., Yamamori, H., Sanada, M., Nishizawa, M., and Okui, K. 1992. Intravenous intralipid $10 \%$ vs. $20 \%$, hyperlipidemia, and increase in lipoprotein $\mathrm{X}$ in humans. Nutrition 8: 155-160.
51. Tomsits, E., Rischak, K., Molnar, M., Filiczky, I., and Szollar, L. 1995. Effects of administration of different intravenous lipid emulsions on plasma LP-X concentrations in the rat. Jpn. J. Parenter. Enteral. Nutr. 19: 369-372.

52. Toyokuni, S., Tanaka, T., Hattori, Y., Yoshida, A., Uchida, K., Hiai, H., Ochi, H., and Osawa, T. 1997. Quantitative immunohistochemical determination of 8-hydroxy-2'deoxyguanosine by a monoclonal antibody N45.1: Its application to ferric nitrilotriacetate-induced renal carcinogenesis model. Lab. Invest. 76: 365-374.

53. Toyokuni, S. 1999. Reactive oxygen species-induced molecular dameges and its application in pathology. Pathol. Int. 49: 91-102.

54. Tozer, E.C. and Carew, T.E. 1997. Residence time of lowdensity lipoprotein in the normal and atherosclerotic rabbit aorta. Circ. Res. 80: 208-218.

55. Vigna, G.B., Bolzan, M., Romagnoni, F., Valerio, G., Vitale, E., Zuliani, G., and Fellin, R. 1992. Lipids and other risk factors selected by discriminant analysis in symptomatic patients with supra-aortic and peripheral atherosclerosis. Circulation 85: 2205-2211.

56. Vlassara, H. 1997. Recent progress in advanced glycation end products and diabetic complications. Diabetes 46: S19S25.

57. Weingand, K.W. and Daggy, B.P. 1990. Quantitation of high-density lipoprotein cholesterol in plasma from hamsters by differential precipitation. Clin. Chem. 36: 575.

58. Wilson, T.A., Meservey, C.M., and Nicolosi, R.J. 1998. Soy lecithin reduces plasma lipoprotein cholesterol and early atherogenesis in hypercholesterolemic monkeys and hamsters: beyond linoleate. Atherosclerosis 140: 147-153.

59. Wu, J.T. 1993. Advanced glycosylation end products: A new disease marker for diabetes and aging. J. Clin. Lab. Anal. 7: 252-255.

60. Yamaguchi, Y., Kagota, S., Kunitomo, M., and Haginaka, J. 1998. Evidence of modified lipoprotein in the plasma of Watanabe heritable hyperlipidemic rabbits by anionexchange high performance liquid chromatographic assay. Atherosclerosis 139: 323-331.

61. Yamamura, T. 1993. Agarose gel electrophoresis. pp. 209213. In: Shin Seikagaku Zikken Kouza/ Neutral lipids and Lipoproteins (Japanese Association of Biochemistry ed.), Tokyo Kagaku Douzin, Tokyo (in Japanese).

62. Yamanouchi, J., Nishida, E., Itagaki, S., Kawamura, S., Doi, K., and Yoshikawa, Y. 2000. Aortic atheromatous lesions developed in APA hamsters with streptozotocininduced diabetes: A new model for diabetic atherosclerosis. 1. Histopathological studies. Exp. Anim. 49 (in press).

63. Yegin, A. and Ozben, T. 1995. Serum glycated lipoproteins in type II diabetic patients with and without complications. Ann. Clin. Biochem. 459-463.

64. Yla, H.S., Palinski, W., Rosenfeld, M.E., Parthasarathy, S., Carew, T.E., Butler, S., Witztum, J.L., and Steinberg, D. 1989. Evidence for the presence of oxidatively modified low density lipoprotein in atherosclerotic lesions of rabbit and man. J. Clin. Invest. 84: 1086-1095. 